\section{Persistent peristomal leakage from percutaneous endoscopic gastrostomy successfully treated with endoscopic suturing}
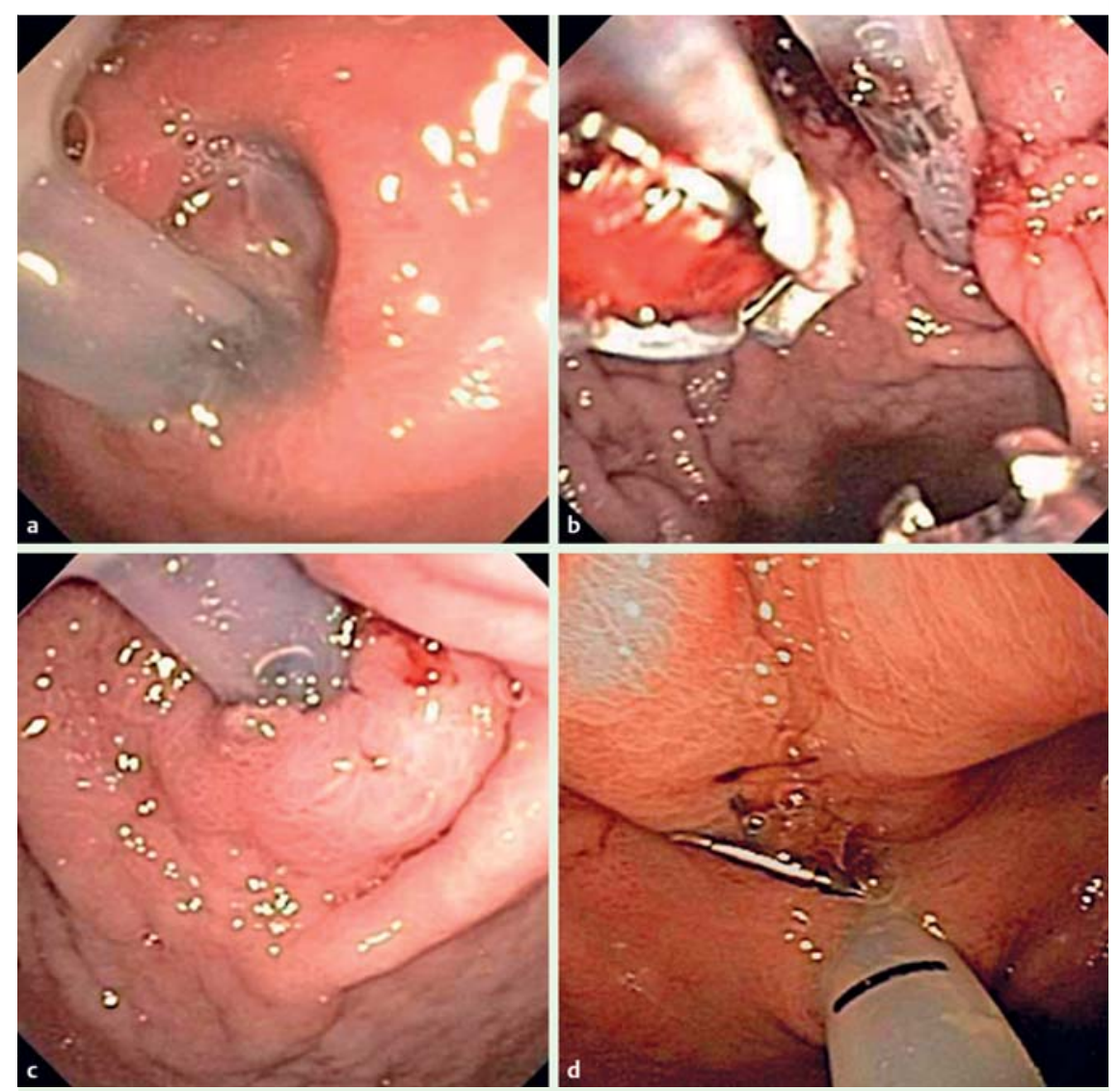

Fig. 1 a Upper gastrointestinal endoscopy showed an enlarged stoma creating a fistulous tract surrounding a percutaneous endoscopic gastrostomy (PEG) tube, leading to air and gastric content leakage and skin maceration. b, c The Overstitch device was used to place a suture and form a 'mucosal wrap' to close the defect and eliminate leakage. $\mathbf{d}$ At 8-week follow-up endoscopy, the wrap was still in place and effective.

We report a novel and successful treatment for persistent peristomal leakage from a recently placed percutaneous endoscopic gastrostomy (PEG) tube, using an endoscopic suturing device.

A 63-year-old man with oral cancer presented with persistent peristomal leakage and severe dermal maceration around a PEG that had been placed 2 weeks previously at an external facility. An initial endoscopy was suboptimal as there was severe leakage of air through the stoma. PEG removal for closure of the stoma needed to be avoided because of the risk of peritonitis given the short time since placement, and aggressive wound care was recommended. Unfortunately, the patient's symptoms worsened over the next week.
We decided to attempt an endoscopic reduction of the stoma tract. Gauze was applied externally to maintain gastric insufflation. Repeat endoscopy revealed an enlarged PEG stoma creating a fistulous tract ( Fig.1a). Following overtube placement, the Overstitch suturing device (Apollo Endosurgery Inc., Austin, Texas, USA) was frontloaded onto a double-channel therapeutic endoscope (GIF-2T160; Olympus Inc; Tokyo, Japan) and inserted into the stomach. The first needle-bite was placed on the upper lip $5 \mathrm{~mm}$ proximal to the defect and the second $5 \mathrm{~mm}$ distally on the lower lip.Utilizing a single continuous suture, a 'mucosal wrap' was fashioned to close the defect ( Fig. 1 b). This eliminated the fistulous tract while allowing adequate movement of the PEG through the stoma ( Fig. 1 c). There was no air leak at the conclusion of the procedure.

The patient reported no leakage at a 2-week follow-up appointment with his oncologist. At an 8-week follow-up endoscopy, the wrap was still in place ( Fig. 1 d) and effective. The patient reported a minimal amount of recurrent output and marked improvement in dermal maceration.

To our knowledge, this is the first report of the use of an endoscopic suturing device to eliminate peristomal leakage from around a PEG. This is a modification of a technique previously described for closure of nonhealing gastrocutaneous fistulas occurring after PEG removal [1,2] The benefit of our method is the alleviation of concern regarding PEG removal from a non-mature tract that could lead to peritonitis. Thus, we favour consideration of our 'mucosal wrap' technique in situations where PEG removal is contraindicated but peristomal leakage is persistent and severe.

Endoscopy_UCTN_Code_TTT_1AO_2AK

Competing interests: None

\section{Peter P. Stanich, Brett Sklaw, Somashekar G. Krishna}

Division of Gastroenterology, Hepatology and Nutrition, Ohio State University Wexner Medical Center, Columbus, Ohio, USA

\section{References}

1 Armengol-Miro JR, Dot J, Abu-Suboh Abadia $M$ et al. New endoscopic suturing device for closure of chronic gastrocutaneous fistula in an immunocompromised patient. Endoscopy 2011; 43 (Suppl. 02): E403-404

2 Kantsevoy SV, Thuluvath PJ. Successful closure of a chronic refractory gastrocutaneous fistula with a new endoscopic suturing device (with video). Gastrointestinal endoscopy 2012; 75: 688-690

\section{Bibliography}

DoI http://dx.doi.org/

10.1055/s-0033-1344870

Endoscopy 2013; 45: E394

(c) Georg Thieme Verlag KG

Stuttgart · New York

ISSN 0013-726X

\section{Corresponding author}

\section{Somashekar G. Krishna, MD, MPH}

Ohio State University Medical Center

395 West 12th Avenue, Suite 200

Columbus, $\mathrm{OH} 43210$

USA

Fax: +01-614-293-8518

somashekar.krishna@OSUMC.edu 